\title{
Determinación de factores asociados al hábito de fumar en una población de mujeres en embarazo
}

\author{
Edgar Alfonso Varela*; Juan Carlos Martínez, Martha Lucía Cadena**; Humberto Reynales***
}

\begin{abstract}
RESUMEN: EI consumo de cigarrillo durante el embarazo ocasiona graves daños en el feto; se conoce que el hábito es mayor entre las maternas de más edad, en multíparas, en madres solteras y en estratos con pobre nivel de educación. Se realizó un estudio piloto para conocer la prevalencia del consumo de cigarrillo en maternas y describir las variables que reseña la literatura como factores asociados con este hábito. Se analizó una población de 50 maternas en el Hospital de San José en Bogotá, encuestándolas sobre su hábito de fumar, número de cigarrillos consumidos, variables demográficas y conocimiento de los efectos nocivos del consumo de cigarrillo durante el embarazo. Se obtuvieron frecuencias absolutas y relativas para cada variable, se utilizó la prueba de t para comparar medias y proporciones y se obtuvieron valores de p. El 14\% de las maternas eran fumadoras, seis de ellas dejaron de fumar durante el embarazo. Se encontró un promedio de edad mayor en el grupo de fumadoras $(p=0.011)$. Todas las maternas no fumadoras eran casadas $(p=0.013)$. Un mayor número de no fumadoras cree que el hábito ocasiona parto prematuro. La ampliación de este estudio acrecentará el conocimiento de este problema de salud en nuestra comunidad para facilitar el desarrollo de nuevas estrategias educativas.
\end{abstract}

PALABRAS CLAVES: Consumo de cigarrillo durante el embarazo.

SUMMARY: Smoking during pregnancy can produce severe damage to the fetus. It is known the habit is more frequent in older, multiparous, nonmarried and lower education level mothers. A pilot study to define the prevalence of smoking during pregnancy and to describe the associated factors. 50 pregnat women at the Hospital de San José in Bogotá, Colombia were surveyed abot smoking habit, demographic variables and knowledge of nocives effects in the fetus.The $t$ test was used to compare proportions and means. The $14 \%$ of the patients smoked and six out of seven stoped the habit during the pregnancy. The group of smokers had a higher age mean ( $p=0.011)$ and all the nonsmokers were married $(p=0.013)$. To develop this study will help to increase the knowledge about this important problem in public health.

KEY WORDS: Smoking during pregnancy.

\section{Introducción}

El hábito de fumar ocasiona graves trastornos en la salud de los individuos. Durante el embarazo estos daños ocurren en la madre y en el feto. Los recién nacidos de madres fumadoras tienen un mayor riesgo de parto pretérmino, de nacer con bajo peso y sufren de una mayor mortalidad perinatal (1-5).

El riesgo de bajo peso al nacer se asocia en especial con el consumo durante el segundo y tercer trimestre, depende de la cantidad de cigarrillos consumidos $(3,6-7)$ y es mayor entre las maternas de mayor edad (2) y en multíparas (8).

La literatura muestra que el consumo de cigarrillo durante el embarazo es mayor en madres no casadas, en poblaciones con niveles menores de educación y para algunos estudios, en las comunidades de raza negra $(4,9)$.

Este estudio se planteó como una investigación piloto para conocer la prevalencia del consumo de cigarrillo en

\footnotetext{
* Médico Internista. Cardiólogo. Instructor Facultad de Medicina. Universidad del Rosario. Santa Fe de Bogotá.

** Estudiantes. Facultad de Medicina. Universidad del Rosario.

*** Médico Internista. Epidemiólogo. Instructor Asociado. Facultad de Medicina. Universidad del Rosario.
}

maternas y para describir algunas características asociadas con el hábito de fumar en el embarazo. Los estudios que han evaluado la tasa de abandono del hábito de fumar durante el embarazo muestran resultados variables (4-5). El conocimiento de los factores que rodean el consumo durante el embarazo, permitirá ejecutar adecuados planes de salud que logren incentivar a la mujer para que abandone su hábito durante el embarazo y para que no lo reanude después.

\section{Materiales y métodos}

Se realizó un estudio piloto en el segundo semestre de 1993 eligiendo al azar una población conformada por 50 maternas que asistieron al servicio de Obstetricia del Hospital de San José en Santafé de Bogotá, un hospital Universitario con un área de influencia en la zona centro y sur-occidental dela ciudad.

Las variables dependientes fueron el hábito de fumar, el número de cigarrillos consumidos al día y los cambios en el hábito durante el embarazo, según si disminuyó, aumentó o permaneció igual. Se evaluaron las siguientes variables independientes edad, estado civil, ocupación, nivel de educación, ingreso familiar, número de gestaciones, aceptación del embarazo, presencia de fuma- 
dores en el núcleo familiar y conocimiento de los efectos nocivos del consumo de cigarrillo durante el embarazo, a través de una encuesta previamente diseñada.

Se usó el programa EPI-INFO versión 6.0 como base de datos y para el análisis estadístico. Se obtuvieron frecuencias absolutas y relativas para cada variable en los grupos de fumadoras y no fumadoras. Se utilizó la prueba de t para comparar las medias y proporciones y se obtuvieron valores de $\mathrm{p}$ con una significancia del $95 \%$.

\section{Resultados}

El rango de edad de la población analizada era de 15 a 50 años. En la Tabla 1 se muestran las características de la población estudiada. El $88 \%$ de las maternas tenían embarazo deseado, el $82 \%$ cursaban su noveno mes de embarazo y el $12 \%$ el octavo mes. Se encontraron 7 maternas (14\%) fumadoras, con un consumo promedio de 1.4 cigarrillos al día y todas ellas con un hábito de más de cinco años. De estas

\section{Tabla 1 \\ CARACTERISTICAS DEMOGRAFICAS DE LA POBLACION ESTUDIADA. HOSPITAL DE SAN JOSE, SANTA FE DE BOGOTA}

\begin{tabular}{|c|c|c|}
\hline Característica & $\mathrm{n}=50$ & $\%$ \\
\hline \multicolumn{3}{|l|}{ Edad } \\
\hline $15-24$ & 16 & 32 \\
\hline $25-34$ & 26 & 52 \\
\hline $35-44$ & 6 & 12 \\
\hline $45-54$ & 2 & 4 \\
\hline \multicolumn{3}{|l|}{ Estado civil } \\
\hline Soltera & 2 & 4 \\
\hline Casada & 23 & 46 \\
\hline Unión libre & 25 & 50 \\
\hline \multicolumn{3}{|l|}{ Ocupación } \\
\hline Hogar & 31 & 62 \\
\hline Empleada & 12 & 24 \\
\hline Secretaria & 4 & 8 \\
\hline Otro & 3 & 6 \\
\hline \multicolumn{3}{|l|}{ Educación } \\
\hline Primaria & 19 & 38 \\
\hline Bachillerato & 28 & 56 \\
\hline Universidad & 3 & 6 \\
\hline \multicolumn{3}{|l|}{ Vivienda } \\
\hline Propia & 8 & 16 \\
\hline Arrendada & 41 & 82 \\
\hline Otro & 1 & 2 \\
\hline \multicolumn{3}{|l|}{ Ingresos* } \\
\hline Menos de 1 & 2 & 4 \\
\hline $1-2$ & 39 & 78 \\
\hline $3-4$ & 7 & 14 \\
\hline $5-6$ & 1 & 2 \\
\hline Más de 6 & 1 & 2 \\
\hline
\end{tabular}

* De acuerdo con el número de salarios mínimos.
Tabla 2

ANTECEDENTES GINECO-OBSTETRICOS DE LOS GRUPOS EN ESTUDIO

\begin{tabular}{|lcccccr|}
\hline & & \multicolumn{3}{c}{ Fumadoras } & \multicolumn{3}{c|}{ No fumadoras } \\
& & No. & $\%$ & \multicolumn{1}{c}{ No. } & \% Valor p \\
\hline EMBARAZOS & 1 & 2 & 28.6 & 19 & 44.2 & 0.65 \\
& 2 & 1 & 14.3 & 14 & 32.6 & 0.53 \\
& 3 & 2 & 28.6 & 6 & 14 & 0.52 \\
& 4 & 0 & & 3 & 7 & $* *$ \\
& 5 & 1 & 14.3 & 1 & 2.3 & NS \\
& 6 & 0 & & 0 & & $* *$ \\
& 9 & 1 & 14.3 & 0 & & $* *$ \\
PARTOS & Promedio & 3.43 & & 1.91 & & 0.27 \\
& 1 & 2 & 28.6 & 22 & 51.1 & 0.44 \\
& 2 & 1 & 14.3 & 15 & 34.9 & 0.46 \\
& 3 & 2 & 28.6 & 4 & 9.3 & NS \\
& 4 & 1 & 14.3 & 1 & 2.3 & NS \\
& 5 & 0 & & 1 & 2.3 & $* *$ \\
TIEMPO & 6 & 1 & 14.3 & 0 & & $* *$ \\
DE EMB. & Promedio & 2.86 & & 1.67 & & 0.23 \\
& 2 & 0 & 1 & 2.3 & & $* *$ \\
& 5 & 0 & 2 & 4.7 & & $* *$ \\
& 8 & 0 & 6 & 14 & & $* *$ \\
& 9 & 7 & 100 & 34 & 79.1 & \\
& Promedio & 8.51 & & 8.58 & & 0.89 \\
\hline
\end{tabular}

* Estadísticamente significativo

** Indeterminado

NS: No significativo

siete, seis dejaron de fumar en razón a su embarazo y sólo una aumentó el hábito, en esta última paciente, el embarazo no era deseado; y aunque la diferencia no es estadísticamente significativa por el escaso número de pacientes, este hecho llama la atención.

La comparación entre el grupo de mujeres fumadoras y las no fumadoras se muestra en la Tabla 2. Se encontró una diferencia estadística significativa en la comparación de los promedios de edad para los dos grupos, siendo mayor en las fumadoras. En el estado civil también se encontró diferencia, pues todas las fumadoras estaban casadas. No existió diferencia estadística significativa de acuerdo con la ocupación, nivel de educación, número de personas que fumaran en el núcleo familiar, el hecho de poseer o no vivienda propia, el nivel de ingreso salarial, el número de gestaciones ni el de partos. En cuanto a la asociación con el conocimiento acerca del riesgo para el recién nacido existió diferencia entre los dos grupos sólo para prematurez, pues un número mayor de no fumadoras cree que el hábito ocasiona parto prematuro $(\mathrm{p}=0.029)$. Tabla 3.

\section{Discusión}

La evidencia clínica de las lesiones que ocasiona el hábito de fumar son claras; aún así, algunas mujeres fuman durante el embarazo.

Se ha descrito la asociación del hábito de fumar durante el embarazo con algunos factores. Al igual que la literatura encontramos un promedio mayor de edad 
Tabla 3

DISTRIBUCION DE FUMADORES EN LA FAMILIA, Y CREENCIAS SOBRE EFECTOS DEL CIGARRILLO

EN EL RECIEN NACIDO

\begin{tabular}{|llrrrrr|}
\hline & \multicolumn{3}{c}{ Fumadoras } & \multicolumn{3}{c|}{ No fumadoras } \\
& & No. & \% & \multicolumn{1}{c|}{ No. } & \% & Valor p p \\
\hline FUMADORES & 0 & 3 & 42.9 & 27 & 64.3 & 0.43 \\
EN LA FAMILIA & 1 & 3 & 42.9 & 10 & 23.8 & 0.4 \\
& 2 & 1 & 14.3 & 5 & 11.9 & NS \\
Promedio & & 0.71 & & 0.47 & & 0.41 \\
BAJO PESO & $\mathrm{Si}$ & 6 & 85.7 & 22 & 52.4 & 0.2 \\
& No & 1 & 14.3 & 20 & 47.6 & \\
PREMATURO & $\mathrm{Si}$ & 6 & 85.7 & 15 & 35.7 & 0.029 \\
& $\mathrm{No}$ & 1 & 14.3 & 27 & 64.3 & \\
RESPIRATORIO & $\mathrm{Si}$ & 6 & 85.7 & 33 & 78.6 & 0.8 \\
& $\mathrm{No}$ & 1 & 14.3 & 9 & 21.4 & \\
\hline
\end{tabular}

* Estadísticamente significativo

** Indeterminado

NS: No significativo entre las embarazadas que fumaban (2) . Esto puede representar un descenso en el hábito de fumar entre la población más joven o por el contrario una pérdida de conciencia, con la edad, respecto a no fumar antes del parto. Aparentemente encontramos una mayor asociación entre el consumo y las mujeres casadas, que es contrario a lo descrito por la literatura $(4,9)$; $\sin$ embargo, el tamaño de la muestra no permite esclarecer si esta misma diferencia exista, en nuestro trabajo, entre el grupo de solteras y de unión libre. No se encontró diferencia entre el ingresosalarial y el hábito. Como la población estudiada se concentraba en el grupo de ingresos entre uno y cinco salarios mínimos, no podemos decir al igual que la literatura si existe cambio del hábito de acuerdo con el nivel de ingresos; pero entre estos rangos no existió diferencia.

Con este trabajo piloto se plantea la necesidad de realizar un estudio para conocer la prevalencia del consumo de cigarrillo durante el embarazo confirmando el consumo con mediciones de metabolitos de nicotina, pues es sabido que el $38 \%$ de las madres expuestas contestan las encuestas como no fumadoras. De otra parte el análisis de los factores asociados con el hábito permitirá diseñar mejores alternativas educativas.

\section{BIBLIOGRAFIA}

1. Wilcox AJ. Birth weight and perinatal mortality: The effect of maternal smoking. Am. J. Epidemiol. 1993; 137(10): 1098-1104.

2. Fox SH., Koepsell TD and Daling JR. Birth weight and smoking during pregnancy - Effect modification by maternal age. Am J Epidemiol 1994; 139(10): 1008-1015.

3. Bardy $\mathrm{AH}$ et al. Objectively measured tobacco exposure during pregnancy: neonatal effects and relation to maternal smoking. Br. J. Obstet. Gynecology. 1993; 100: 721-726.

4. Mittendorf R et al. Reducing the frequency of low birth weight in the United States. Obstet. Gynecol. 1994; 83: 1056-1059.

5. Li C.Q et al. The impact on infant birth weight and gestational age of cotinine-validated smoking reduction during pregnancy. JAMA. 1993; 269: 1519-1524.
6. Lieberman $\mathrm{E}$ et al. Low birthweight at term and the timing of fetal exposure to maternal smoking. Am. J. Public. Health 1994; 84: 1127 1131.

7. Wilcox $\mathrm{M}$ et al. Birth weight from pregnancies dated by ultrasonography in a multicultural British population. BMJ. 1993; 307: $588-591$

8. Cnattingius $S$ et al. Effect of age, parity, and smoking on pregnancyoutcome: a population-based study. Am. J. Obstet. Gynecol. 1993; 168: 16-21.

9. Land GH and Stockbauer JW. Smoking and pregnancy outcome: trends among black teenage mothers in Missouri. Am. J. Public Health 1993; 83: 1121-1124. 